\title{
Capsaicin-induced metabolic and cardiovascular autonomic improvement in an animal model of the metabolic syndrome
}

\author{
Camila da Silva Tremarin ${ }^{1,2 *}$, Karina Rabello Casali ${ }^{1}$, Luise Meurer ${ }^{3}$ and Beatriz D'Agord Schaan ${ }^{1,2}$ \\ ${ }^{1}$ Instituto de Cardiologia/Fundação Universitária de Cardiologia, Porto Alegre, Brazil \\ ${ }^{2}$ Endocrine Division, Hospital de Clinicas de Porto Alegre, Universidade Federal do Rio Grande do Sul, Porto Alegre, Brazil \\ ${ }^{3}$ Pathology Division, Hospital de Clinicas de Porto Alegre, Universidade Federal do Rio Grande do Sul, Porto Alegre, Brazil \\ (Submitted 20 September 2012 - Final revision received 2 July 2013 - Accepted 2 July 2013 - First published online 21 August 2013)
}

\section{Abstract}

The metabolic syndrome (MetS) is associated with an increased risk of cardiac mortality, as it is characterised by the clustering of multiple cardiovascular risk factors. Studies have shown that capsaicin (red pepper) may be useful as a nutraceutical, ameliorating metabolic profile and cardiovascular function. The aim of the present study was to investigate the cardiovascular and metabolic effects of orally administered capsaicin in rats with the MetS. Neonate spontaneously hypertensive rats were injected with monosodium glutamate and subjected to one of the following three treatments by oral administration for $14 \mathrm{~d}$, between 27 and 30 weeks: low-dose capsaicin (CAP05, $n$ 18, synthetic capsaicin powder diluted in a vehicle ( $10 \%$ ethyl alcohol) plus $0.5 \mathrm{mg} / \mathrm{kg}$ body weight (BW) of capsaicin); high-dose capsaicin (CAP1, $n$ 19, synthetic capsaicin powder diluted in a vehicle ( $10 \%$ ethyl alcohol) plus $1 \mathrm{mg} / \mathrm{kg}$ BW of capsaicin); control (C, $n$ 18, vehicle). Lee's index, lipid/metabolic profile, and cardiovascular parameters with the rats being conscious, including arterial pressure (AP) and heart rate (HR) variability, as well as aortic wall thickness (haematoxylin and eosin staining) and CD68 (cluster of differentiation 68) antibody levels (monocyte/macrophage immunostaining) were evaluated. Weight, Lee's index, and lipid and metabolic parameters, as well as AP and HR and aortic wall thickness, were similar between the groups. Capsaicin determined HR variability improvement (16.0 (SEM 9.0), 31.0 (SEM 28.2) and 31.3 (SEM 19.0) $\mathrm{ms}^{2}$ for the C, CAP05 and CAP1 groups, respectively, $P=0 \cdot 003$ ), increased vascular sympathetic drive (low-frequency component of systolic AP variability: $3 \cdot 3$ (SEM 2.8), $8 \cdot 2$ (SEM 7.7) and 12.1 (SEM $8 \cdot 8$ ) mmHg ${ }^{2}$ for the C, CAP05 and CAP1 groups, respectively, $P<0 \cdot 001)$ and increased $\alpha$-index (spontaneous baroreflex sensitivity). The present data show that capsaicin did not improve lipid and glucose abnormalities in rats with the MetS. However, beneficial cardiovascular effects were observed with this nutraceutical.

Key words: Metabolic syndrome X: Capsaicin: Cholesterol: Blood glucose: Autonomic nervous system: Blood pressure

The metabolic syndrome (MetS) is a highly prevalent condition where multiple cardiovascular risk factors aggregate, which is associated with increases in cardiovascular morbidity and mortality ${ }^{(1)}$. Studies in animal models can be relevant as they mimic the aspects of the human disease regarding the development and maintenance of the characteristics of the MetS, particularly obesity, type 2 diabetes, dyslipidaemia and hypertension. Experimental models of the MetS may be genetic, chemically induced or diet induced ${ }^{(2)}$. The neonate spontaneously hypertensive rat (SHR) subcutaneously administered monosodium glutamate is an animal model that presents a phenotype similar to that of the MetS that occurs in humans, making it useful for its study, as has been recently shown in our laboratory. SHR present several characteristics (obesity, hyperglycaemia, insulin resistance, hypertriacylglycerolaemia, reduced HDL-cholesterol levels and hypertension) of the MetS. Furthermore, reduced GLUT4 content in insulin-sensitive tissues and increased plasma concentration of inflammatory markers have been reported ${ }^{(3)}$.

Due to the lack of a single drug for the treatment of the MetS, there is growing interest in the study of alternative substances, such as nutraceuticals. Green tea ${ }^{(4)}$ and curcumin ${ }^{(5)}$ are among some of the phytochemicals introduced in the diet as interventions that had been tested and had shown some benefits in the MetS. Capsaicin (8-methyl- $N$-vanillyl-6nonenamide) is one of the most abundant capsaicinoid types present in red pepper fruit from the genus Capsicum, which belongs to the family Solanaceae ${ }^{(6)}$. Capsaicin is also responsible for the pungency of hot red pepper and other Capsicum plants and has shown multiple health-related benefits (protection against LDL oxidation by lowering oxidative stress and increases in diet-induced thermogenesis and lipid

Abbreviations: BW, body weight; HF, high frequency; LF, low frequency; MetS, metabolic syndrome; SAP, systolic arterial pressure; SAPV, systolic arterial pressure variability; SHR, spontaneously hypertensive rats.

*Corresponding author: C. d. S. Tremarin, fax +55 513359 8127, email catremarin@hotmail.com 
oxidation) in both animal studies and human trials ${ }^{(7,8)}$. These benefits could account for its anti-inflammatory properties ${ }^{(9)}$ and effects on diet-induced thermogenesis ${ }^{(8)}$ and lipid profile ${ }^{(10)}$. The daily intake of capsaicinoids has been estimated as $25-200 \mathrm{mg} /$ person per $\mathrm{d}^{(11)}$. However, the acute and chronic effects of capsaicin on glycaemia and insulin resistance, main characteristics of the MetS, have not been studied yet. Blood pressure reduction has been reported to be related to its use $^{(12)}$, but some studies have observed high blood pressure effects $^{(13)}$. The use of capsaicin in an animal model that clusters many of the features of the MetS, as is SHR treated with monosodium glutamate, could throw light on these inconsistencies.

Considering these controversies and that most studies have evaluated the effects of capsaicin after acute administration, it is not possible to recommend the use of capsaicin. The aim of the present study was to investigate metabolic and cardiovascular changes induced by chronic oral administration of capsaicin in an animal model of the MetS.

\section{Experimental methods}

The investigation followed the ethical rules established by the Guide for the Care and Use of Laboratory Animals ${ }^{(14)}$ and the ARRIVE (Animal Research: Reporting In vivo Experiments) guidelines on animal research ${ }^{(15)}$. The present study was approved by the Research Ethics Committee of our institution.

\section{Animals}

Neonate male SHR ( $n$ 55) were subjected to the subcutaneous administration of monosodium glutamate $(5 \mathrm{mg} / \mathrm{g}$ per $\mathrm{d}$; Sigma Chemical Company) diluted in a saline solution ( $0.9 \%$ $\mathrm{NaCl}$ ) for nine consecutive days; after $21 \mathrm{~d}$, the rats were weaned and placed into plastic boxes, four rats per box. The rats were bred and kept under standard laboratory animal housing conditions at the Animal Production and Research Unit at our institution, receiving standard laboratory chow and tap water ad libitum. They were exposed to a $12 \mathrm{~h}$ light and $12 \mathrm{~h}$ dark cycle (06.00 hours/18.00 hours).

At 6 months of age, the rats were randomised into controls, which received a regular diet ( $C, n$ 18); a low-dose capsaicin group, which received a regular diet plus $0.5 \mathrm{mg} / \mathrm{kg}$ body weight (BW) of capsaicin (CAP05, $n$ 18); and a high-dose capsaicin group, which received a regular diet plus $1 \mathrm{mg} / \mathrm{kg}$ BW of capsaicin (CAP1, $n$ 19). The capsaicin used was synthetic $N$-vanillylnonanamide, with a chemical structure similar to that of pure capsaicin (Sigma Chemical Company), diluted in $10 \%$ ethyl alcohol $(20 \mathrm{ml}$ of ethyl alcohol in $200 \mathrm{ml}$ of water) and administered daily $(2 \mathrm{ml} / \mathrm{kg} \mathrm{BW})$ at a concentration of 0.25 or $0.5 \%$ for $14 \mathrm{~d}$ via orogastric administration. The controls received the same amount of an alcohol vehicle solution during the same period.

\section{Metabolic evaluation}

Weight and Lee's index (cube root of BW in g divided by noseto-anus length in $\mathrm{cm})^{(16)}$ were evaluated before and after the protocols.
At the end of the treatment with capsaicin or vehicle, all the rats were placed into individual metabolism cages for $24 \mathrm{~h}$. An insulin tolerance test was performed the next morning using commercially available regular insulin (Biohulin; Biobrás). The rats were anaesthetised (sodium thiopental, intraperitoneally) after $3 \mathrm{~h}$ of food deprivation, and then insulin $(0.75 \mathrm{U} / \mathrm{kg} ; 5.4 \mathrm{pmol} / \mathrm{kg})$ was administered into the penile vein. Later, six blood glucose measurements (Accu-Chek Advantage; Roche) were taken: at baseline and 4, 8, 12, 16 and $20 \mathrm{~min}$ after insulin administration. Glycaemias were then converted into the natural logarithm (Ln); the slope was calculated using linear regression (time $\times$ Ln (glucose)) and multiplied by 100 to obtain the glucose decay constant rate during the insulin tolerance test per $\min (\% / \mathrm{min})^{(17)}$.

The rats were anaesthetised $(90 \mathrm{mg} / \mathrm{kg}$ ketamine and $10 \mathrm{mg} / \mathrm{kg}$ xylazine, intraperitoneally) on the next day to implant a catheter filled with heparinised saline into the femoral artery for the direct measurements of arterial pressure. The end of the catheter was tunnelled subcutaneously and exteriorised at the top of the head. Cardiovascular evaluation was performed $24 \mathrm{~h}$ after the placement of the catheter.

\section{Cardiovascular evaluation}

The rats were conscious in their cages and allowed to move freely during the experiments. The catheter was connected to a transducer (P23Db; Gould-Statham), and arterial pressure signals were recorded for a $30 \mathrm{~min}$ period using a microcomputer equipped with an analogue-to-digital converter (CODAS, 2Kz; DATAQ Instruments). The recorded data were analysed on a beat-to-beat basis to quantify changes in systolic arterial pressure (SAP), diastolic arterial pressure and mean arterial pressure and heart rate.

\section{Assessment of autonomic control - spectral analysis}

The time series of pulse intervals (tachograms) and SAP (systograms) were obtained from arterial pressure records through the beat-to-beat pulse intervals and SAP values, respectively. Stationary fragments with about 300 beats, coincident in tachograms and systograms, were selected and a spectral analysis was performed using an autoregressive model. The spectral bands for rats (very low frequency (LF): 0.0-0.2 Hz; LF: $0 \cdot 2-0.75 \mathrm{~Hz}$; high frequency $(\mathrm{HF}): 0 \cdot 75-3 \cdot 0 \mathrm{~Hz})$ were defined as described previously ${ }^{(18)}$. Tachogram and systogram spectra for each stationary fragment were evaluated quantitatively, obtaining the values of heart rate variability and SAP variability (SAPV). The spectral components of pulse intervals and SAP are expressed in both absolute ( $\mathrm{ms}^{2}$ and $\mathrm{mmHg}^{2}$, respectively) and normalised units. These normalised units were obtained by calculating the power of LF and HF components and correlating them to the total power without the very-LF component ${ }^{(18,19)}$. Spectral decomposition estimates the centre frequency and power of each band associated with sympathetic/parasympathetic systems in the cardiovascular control. The ratio of LF:HF components was related to cardiac sympathovagal balance. Very-LF and LF components from SAPV were associated with renin-angiotensin and vascular sympathetic modulations, 
respectively. The $\alpha$-index was obtained from the square root of the ratio of the R-R interval to the SAPV within the LF band, calculated only when the coherence of the LF band exceeded $0 \cdot 5$. This index was used to estimate the spontaneous baroreflex sensitivity ${ }^{(18-21)}$

The rats were again anaesthetised with the same amounts of anaesthetics mentioned above. Blood samples were drawn by cardiac puncture after $5 \mathrm{~h}$ of food deprivation. After centrifugation, the sera were aliquoted and frozen until assayed for glycaemia and TAG, cholesterol and HDL-cholesterol concentrations (enzymatic method; commercial kit Labtest); LDL-cholesterol concentration was calculated using the Friedewald formula ${ }^{(22)}$.

\section{Immunohistochemistry}

Rats were killed by exsanguination; the thoracic aorta was removed, dissected and fixed in formalin and embedded in paraffin. Later, $4 \mu \mathrm{m}$ sections were stained with haematoxylin and eosin. CD68 monoclonal antibodies were used to immunostain monocytes/macrophages (Millipore) at a dilution of 1:200. Staining was performed in the Experimental Pathology Unit, Hospital de Clínicas de Porto Alegre, following a routine protocol (deparaffinisation, rehydration, antigen retrieval, inactivation of endogenous peroxidase and blocking of non-specific reactions). The sections were incubated with primary antibodies for $12 \mathrm{~h}$ at $4{ }^{\circ} \mathrm{C}$, followed by application of the streptavidin-biotin-peroxidase complex (labelled streptavidin-biotin (LSAB), Dako Corporation) and staining with diaminobenzidine (DAB kit; Dako Corporation).

The thickness of the aorta was evaluated using the haematoxylin and eosin-stained sections, with a computerised image analysis (Image-Pro Express version 6.0 for Windows; Media Cybernetics, Inc.). The thickness of the intima and media of the wall assembly of the aorta was measured in pixels for each rat. The SHR ( $n$ 4) and Wistar-Kyoto (normotensive and non-obese, $n 5$ ) groups were also included for comparison with rats with the MetS ( $n$ 9).
The metabolic and haemodynamic/variability parameters and CD68 in monocytes were evaluated by blinded researchers.

\section{Statistical analysis}

All data are expressed as means with their standard errors. Statistical analysis was carried out using the KolmogorovSmirnov test to assess the normal distribution of variables. Data were compared using one-way ANOVA or KruskalWallis test for comparisons of normal or non-normal continuous variables, respectively. Tukey's post hoc test or Dunn's method was used to detect whether differences existed. The differences between the groups were accepted to be significant at $P<0 \cdot 05$.

\section{Results}

Weight, Lee's index and metabolic parameters were not significantly changed by capsaicin treatment. These data are given in Table 1.

Haemodynamic parameters and spectral analysis results are shown in Fig. 1 and Table 2. Mean arterial pressure, SAP and diastolic arterial pressure did not change by capsaicin treatment, but it led to an improvement in heart rate variability, which was higher in the group receiving the lower dose of capsaicin (CAP05; Fig. 1(d)). High-dose treatment with capsaicin (CAP1) was associated with an increase in the LF component of SAPV, indicating an increment of the vascular sympathetic drive (Fig. 1(f)); this was similar for the HF component of SAPV (synchronous with respiratory rate) (Table 2). Both groups receiving capsaicin had an increased $\alpha$-index (spontaneous baroreflex sensitivity), which was more pronounced at higher doses (C: 0.69 (SEM 0.12), CAP05: 1.54 (SEM 0.26) and CAP1: $2 \cdot 23($ SEM 0.59$) \mathrm{s} / \mathrm{mmHg}$ ) (Fig. 1(e)).

Fig. 2 shows representative examples of the histological and immunohistochemical analyses of Wistar-Kyoto rats

Table 1. Metabolic parameters of the rats studied after oral administration of capsaicin (CAP05 and CAP1) or alcohol for $14 \mathrm{~d}^{*}$ (Mean values and standard deviations; medians and 25th and 75th percentiles; number of rats in each analysis)

\begin{tabular}{|c|c|c|c|c|c|c|c|c|c|c|}
\hline & \multicolumn{2}{|c|}{ C } & \multirow[b]{2}{*}{$n$} & \multicolumn{2}{|c|}{ CAP05 } & \multirow[b]{2}{*}{$n$} & \multicolumn{2}{|c|}{ CAP1 } & \multirow[b]{2}{*}{$n$} & \multirow[b]{2}{*}{$P$} \\
\hline & Mean & SD & & Mean & SD & & Mean & SD & & \\
\hline Food intake (g/24h) & $19 \cdot 9$ & 4.7 & 13 & 22.5 & 7.4 & 17 & $22 \cdot 7$ & $7 \cdot 6$ & 17 & 0.494 \\
\hline Water intake $(\mathrm{ml} / 24 \mathrm{~h})$ & $42 \cdot 5$ & $12 \cdot 1$ & 13 & $38 \cdot 8$ & $10 \cdot 1$ & 16 & $38 \cdot 8$ & $10 \cdot 9$ & 16 & 0.581 \\
\hline Diuresis $(\mathrm{ml} / 24 \mathrm{~h})$ & $18 \cdot 6$ & $7 \cdot 1$ & 13 & $19 \cdot 2$ & $5 \cdot 2$ & 17 & $20 \cdot 6$ & 7.5 & 17 & 0.718 \\
\hline Weight (g) & $292 \cdot 0$ & $30 \cdot 2$ & 18 & 293.9 & $31 \cdot 7$ & 18 & 296.8 & 31.6 & 19 & 0.888 \\
\hline Lee's index $(\mathrm{g} / \mathrm{cm})$ & 0.30 & 0.02 & 14 & 0.30 & 0.02 & 18 & 0.30 & 0.02 & 18 & 0.554 \\
\hline Glycaemia $(\mathrm{mmol} / \mathrm{l})$ & $6 \cdot 5$ & 1.5 & 13 & $6 \cdot 6$ & 1.5 & 16 & 7.4 & 1.8 & 16 & 0.852 \\
\hline kITT (\%/min) & $5 \cdot 18$ & 1.97 & 13 & $5 \cdot 01$ & $2 \cdot 12$ & 16 & $5 \cdot 14$ & $2 \cdot 01$ & 16 & 0.970 \\
\hline Total cholesterol $(\mathrm{mmol} / \mathrm{l})$ & 1.4 & 0.5 & 12 & 1.3 & 0.3 & 16 & 1.2 & 0.5 & 15 & 0.433 \\
\hline LDL-cholesterol (mmol/l) & 0.6 & 0.5 & 12 & 0.4 & 0.3 & 15 & 0.5 & 0.3 & 14 & 0.435 \\
\hline HDL-cholesterol (mmol/l) & 0.5 & 0.3 & 12 & 0.6 & 0.3 & 16 & 0.5 & 0.2 & 15 & 0.179 \\
\hline TAG $(\mathrm{mmol} / \mathrm{l})$ & & & & & & & & & & 0.435 \\
\hline$n$ & \multicolumn{2}{|c|}{12} & & \multicolumn{2}{|c|}{16} & \multicolumn{3}{|c|}{15} & & \\
\hline Median & \multicolumn{2}{|c|}{0.7} & & \multicolumn{2}{|c|}{0.6} & \multicolumn{3}{|c|}{0.6} & & \\
\hline 25th-75th percentile & \multicolumn{2}{|c|}{$0.5-0.9$} & & \multicolumn{2}{|c|}{$0.6-0.8$} & \multicolumn{3}{|c|}{$0.4-0.9$} & & \\
\hline
\end{tabular}

C (controls), regular diet plus $10 \%$ ethyl alcohol given orally $(2 \mathrm{ml} / \mathrm{kg}$ body weight (BW)); CAP05 (low-dose capsaicin), regular diet plus $0.5 \mathrm{mg} / \mathrm{kg}$ BW of capsaicin diluted in $10 \%$ ethyl alcohol given orally $(2 \mathrm{ml} / \mathrm{kg} \mathrm{BW}$ ); CAP1 (high-dose capsaicin), regular diet plus $1 \mathrm{mg} / \mathrm{kg} \mathrm{BW}$ of capsaicin diluted in $10 \%$ ethyl alcohol given orally ( $2 \mathrm{ml} / \mathrm{kg} \mathrm{BW})$; $\mathrm{kITT}$, glucose decay constant rate during the insulin tolerance test.

${ }^{*}$ Numbers of rats are not the same because of dropouts. 
(a)

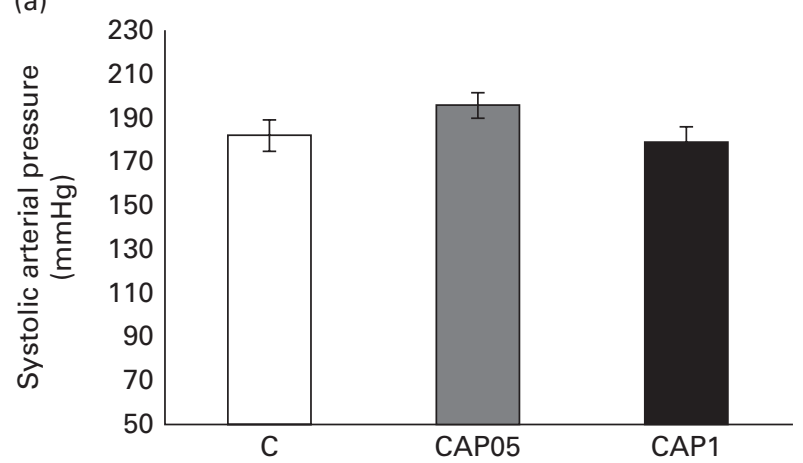

(c)

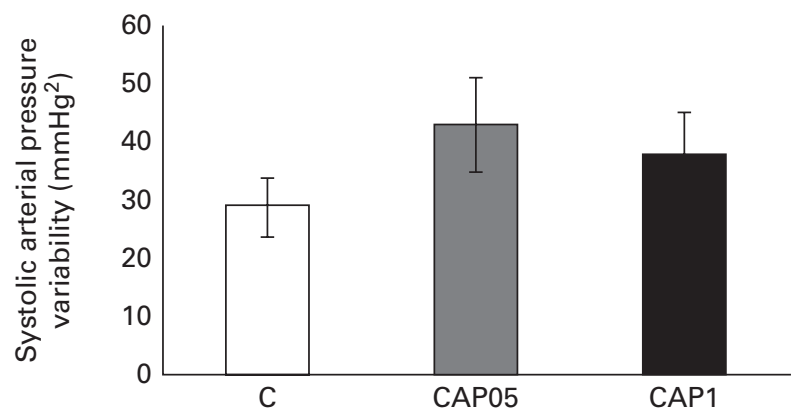

(e)

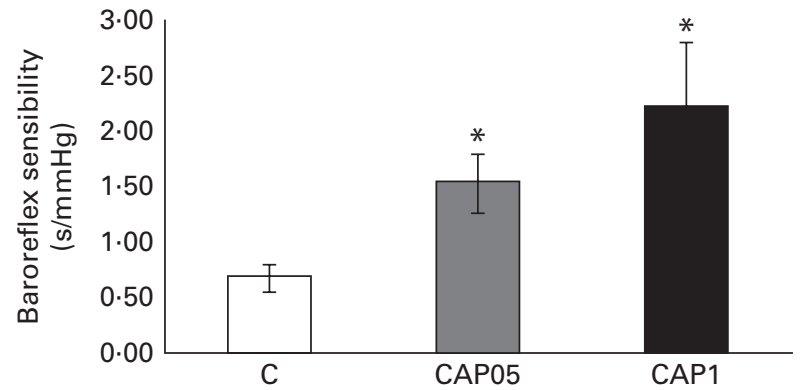

(b)

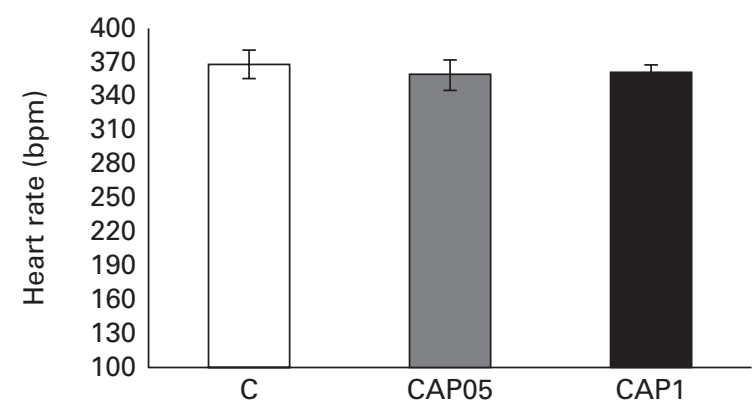

(d)

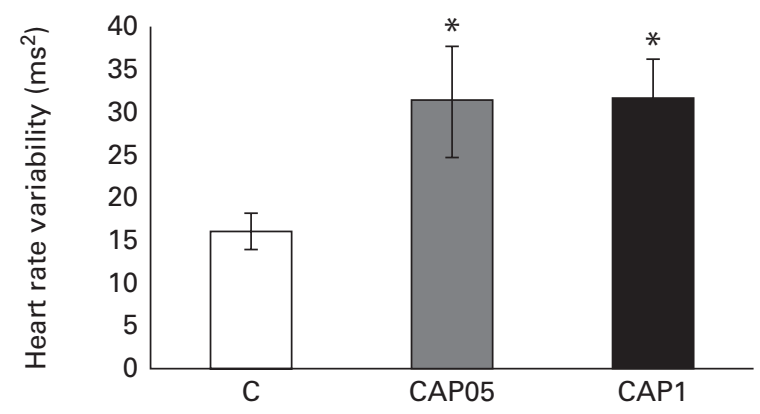

(f)

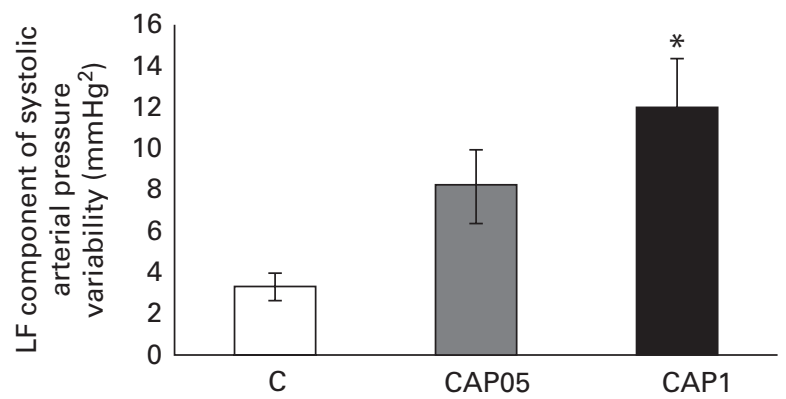

Fig. 1. Cardiovascular autonomic evaluation by spectral analysis. (a) Systolic arterial pressure, (b) heart rate, (c) systolic arterial pressure variability, (d) heart rate variability, (e) spontaneous baroreflex sensibility, related to $\alpha$ low-frequency (LF) index (square root of the ratio of the absolute LF components of heart rate variability and systolic arterial pressure variability), and (f) LF component of systolic arterial pressure. C, control ( $\square$ ), $n$ 17; CAP05, capsaicin $0.5 \mathrm{mg} / \mathrm{kg}$ body weight (BW) daily $(\square), n 18$; CAP1, capsaicin $1.0 \mathrm{mg} / \mathrm{kg} \mathrm{BW}$ daily $(\square), n 16$. Values are means, with their standard errors represented by vertical bars. ${ }^{*}$ Mean value was significantly different from that of the control group $(P<0.05$; one-way ANOVA and Dunn's post hoc test). bpm, Beats per min.

(Fig. 2(a) and (b)), SHR (Fig. 2(c) and (d)) and rats with the MetS (Fig. 2(e) and (f)). Fig. 2(a), (c) and (e) shows representative examples for each group of the haematoxylin and eosin-stained thoracic aorta tissue sections. Fig. 2(b), (d) and (f) shows the immunohistochemical expression of CD68 in the same tissue of representative examples from each group. The comparison between the groups indicated higher aortic wall thickness in the SHR (358.8 (SEM 40.3) pixels) and rats with the MetS (401.5 (SEm 29.7) pixels) $v$. Wistar-Kyoto rats (251.4 (SEM 31.4) pixels) $(P<0 \cdot 001)$. The number of stained cells with CD68 was not different between the groups (number of positive cells/twenty fields: SHR, 21.0 (11.5-28.2); rats with the MetS, $20 \cdot 0(8 \cdot 0-30 \cdot 0)$; Wistar-Kyoto rats, $5 \cdot 0(4 \cdot 0-6 \cdot 0)$, $P=0 \cdot 188)$. There were no differences in aortic wall thickness (358.8 (SEM 40.3), 365.1 (SEM 40.0) and 374.4 (SEM 40.8) pixels for the C, CAP05 and CAP1 groups, respectively, $P=0.718$ ) and the number of stained cells with CD68 in immunohistochemical staining (number of positive cells/twenty fields: $20 \cdot 0$ $(8 \cdot 0-30 \cdot 0), 17 \cdot 0(8 \cdot 0-23 \cdot 0)$ and $8 \cdot 0(4 \cdot 5-25 \cdot 0)$ for the $\mathrm{C}$, CAP05 and CAP1 groups, respectively, $P=0.692)$ between the groups of MetS rats that received capsaicin.

\section{Discussion}

Capsaicin has potential beneficial cardiovascular ${ }^{(10,12)}$ and metabolic ${ }^{(7,8)}$ effects, which could be interesting for the management of the MetS, but the present results indicated that there was no benefit from using it for a short period of time with regard to the metabolic parameters evaluated. However, capsaicin promoted global improvement of cardiovascular autonomic control, which has a well-known role in better cardiovascular prognosis ${ }^{(23,24)}$. 
Table 2. Haemodynamic parameters and spectral analysis results of the rats studied after oral administration of capsaicin (CAP05 and CAP1) or alcohol for $14 \mathrm{~d} \dagger$ (Mean values and standard deviations)

\begin{tabular}{|c|c|c|c|c|c|c|c|}
\hline & \multicolumn{2}{|c|}{$\mathrm{C}(n 17)$} & \multicolumn{2}{|c|}{ CAP05 $(n 18)$} & \multicolumn{2}{|c|}{ CAP1 $(n 16)$} & \multirow[b]{2}{*}{$P$} \\
\hline & Mean & SD & Mean & SD & Mean & SD & \\
\hline MAP $(\mathrm{mmHg})$ & 152.5 & $28 \cdot 2$ & $165 \cdot 2$ & $24 \cdot 6$ & 147.6 & $26 \cdot 2$ & 0.123 \\
\hline $\mathrm{DAP}(\mathrm{mmHg})$ & $124 \cdot 9$ & 28.9 & $135 \cdot 7$ & 24.0 & 118.7 & 25.5 & 0.149 \\
\hline \multicolumn{8}{|l|}{ HRV } \\
\hline LF absolute $\left(\mathrm{ms}^{2}\right)$ & $3 \cdot 1$ & 2.9 & $12 \cdot 2$ & 26.9 & $6 \cdot 2$ & $7 \cdot 6$ & 0.235 \\
\hline LF (NU) & $21 \cdot 3$ & 14.0 & 33.4 & $20 \cdot 2$ & $26 \cdot 7$ & 14.4 & 0.251 \\
\hline HF absolute $\left(\mathrm{ms}^{2}\right)$ & 8.6 & $5 \cdot 2$ & $12 \cdot 4$ & 8.2 & 14.9 & $13 \cdot 9$ & 0.173 \\
\hline $\mathrm{HF}(\mathrm{NU})$ & $77 \cdot 2$ & $12 \cdot 9$ & $65 \cdot 3$ & $19 \cdot 8$ & 71.9 & $14 \cdot 1$ & 0.164 \\
\hline LF:HF ratio & 0.4 & 0.3 & 0.8 & 0.9 & 0.4 & 0.4 & 0.397 \\
\hline SAPV & 9.5 & $9 \cdot 0$ & $16 \cdot 6$ & $17 \cdot 7$ & $7 \cdot 1$ & $10 \cdot 3$ & 0.104 \\
\hline \multicolumn{8}{|l|}{$\operatorname{VLF}\left(\mathrm{mmHg}^{2}\right)$} \\
\hline $\mathrm{HF}\left(\mathrm{mmHg}^{2}\right)$ & 3.6 & $6 \cdot 1$ & $3 \cdot 1$ & 2.4 & $7 \cdot 2^{\star}$ & 7.5 & 0.032 \\
\hline
\end{tabular}

C (controls), regular diet plus $10 \%$ ethyl alcohol given orally $(2 \mathrm{ml} / \mathrm{kg}$ body weight (BW)); CAP05 (low-dose capsaicin), regular diet plus $0.5 \mathrm{mg} / \mathrm{kg} \mathrm{BW}$ of capsaicin diluted in $10 \%$ ethyl alcohol given orally $(2 \mathrm{ml} / \mathrm{kg}$ BW); CAP1 (high-dose capsaicin), regular diet plus $1 \mathrm{mg} / \mathrm{kg} \mathrm{BW}$ of capsaicin diluted in $10 \%$ ethyl alcohol given orally $(2 \mathrm{ml} / \mathrm{kg} \mathrm{BW})$; MAP, mean arterial pressure; DAP, diastolic arterial pressure; HRV, heart rate variability; LF, low-frequency component; NU, normalised units; HF, high-frequency component; SAPV, systolic arterial pressure variability; VLF, very-low-frequency component.

* Mean value was significantly different from that of the control group $(P<0.05$; one-way ANOVA and Dunn's method).

t $n$ 16-19 for each group, except for the analysis of MAP and DAP. The numbers of rats evaluated for MAP and DAP were eighteen (C), eighteen (CAP05) and nineteen (CAP1). The spectral parameters of heart rate (tachograms) and systolic arterial pressure (systograms) were calculated from the time series using an autoregressive model.

The doses of capsaicin used did not influence food/water intake and weight during the short period of the experiments, showing that in 2 weeks the rats exhibited good acceptance of the substance, which did not affect their welfare. These results are consistent with previous findings ${ }^{(25)}$, which showed that even when the supplement was added to the diet, its pungent flavour did not affect its palatability. However, mice fed a high-fat diet had reduced BW with no change in food intake $^{(26)}$, suggesting that capsaicin can increase diet-induced thermogenesis, as has been shown with acute administration ${ }^{(8)}$.

The lipid profile also did not change by capsaicin use. Studies in dyslipidaemic ${ }^{(10)}$ or obese mice ${ }^{(26)}$ fed a high-fat diet supplemented with capsaicin showed reductions in serum TAG levels. However, no decrease in plasma cholesterol levels was observed ${ }^{(27)}$, as has also been shown in the present study. In longer-period treatments ${ }^{(7,10)}$, capsaicin added to a hypercholesterolaemic or high-fat diet led to a reduction in serum cholesterol levels. We presume that there is thermogenic inefficiency in the animal model of the MetS, in which capsaicin does not have the same effect on the lipid profile as has been shown successfully in other studies. It is possible that in the combination of obesity ${ }^{(28)}$ and hypertension $^{(29)}$, an impaired metabolism occurs because of reduced amounts of brown adipose tissue, responsible for thermogenic activity. Moreover, it is known that the amounts of this specialised tissue decrease with age, and other authors $^{(7,25,27)}$ have used animals younger than those used in the present study. Although the amounts of brown adipose tissue in humans are low, this tissue is, in fact, very active. Defined regions of functionally active brown adipose tissue have been reported to be present in adult humans. Its amount is inversely correlated with BMI, suggesting a role of brown adipose tissue in the metabolism of adult humans ${ }^{(30,31)}$.

No changes in blood glucose levels and insulin resistance were induced by capsaicin. Other authors have shown that capsaicin increased glycaemia regardless of insulin levels, but evaluations have been performed acutely during the course of the oral glucose tolerance test in healthy subjects ${ }^{(32)}$. Similar to our findings, it has been reported that regular consumption of chilies did not change glycaemia in human subjects $^{(33)}$.

Recently, in the same animal model of the MetS, we have shown that autonomic dysfunction occurred over time, as we observed increased sympathovagal imbalance in MetS rats, as well as changes in SAPV. Autonomic dysfunction accompanied increased visceral fat and insulin resistance in these rats, providing information that cardiovascular autonomic modulation dysfunction evolves over time and is associated with higher amounts of visceral fat and insulin resistance, as observed in humans. Since cardiovascular autonomic dysfunction is associated with a high risk of incident cardiovascular events and death, these data support the use of this animal model in investigations into the pathophysiology of the MetS, especially that related to the cardiovascular system and its neural control ${ }^{(34)}$.

An overall improvement in the autonomic control of SAP with increased heart rate variability and spontaneous baroreflex sensitivity was shown by the spectral analysis. Previous data indicated contrary results, but the animals studied were healthy and assessed after an acute oral administration of capsaicin, showing increases in heart rate and LF:HF ratio, supporting an increase in the sympathetic drive ${ }^{(35)}$. Other 


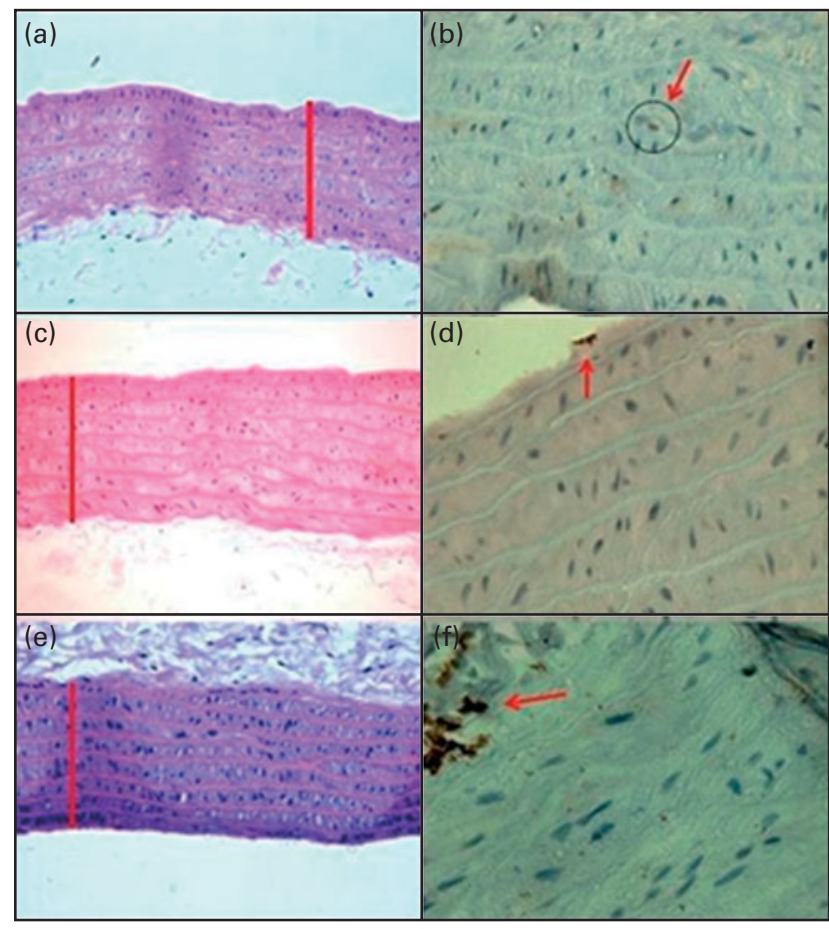

$(\mathrm{g})$

Aortic wall thickness

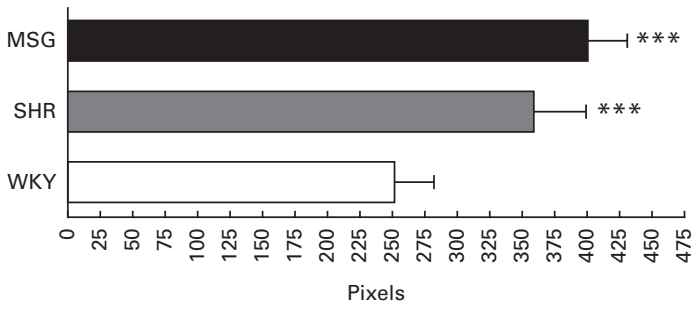

Fig. 2. Histology and immunohistochemistry (Wistar-Kyoto (WKY, $\square$ ) rats, $n 5$; spontaneously hypertensive rats (SHR, $\square$ ), $n 4$; SHR injected with monosodium glutamate (MSG, $\square$ ), $n 9$ ). Values are means, with their standard errors represented by horizontal bars. ${ }^{* \star}$ Mean value was significantly different from that of the WKY rats $(P<0.001)$. (a) WKY rats, (c) SHR and (e) SHR injected with MSG: haematoxylin and eosin-stained thoracic aorta tissue sections of representative examples from each group. Red line marks the thickness of the aorta, which was not significantly different among the groups. Original magnification 400x. (g) A scale bar comparing the aortic wall thickness among the groups. (b) WKY rats, (d) SHR and (f) SHR injected with MSG: immunohistochemical expression of CD68 in the same tissue of representative examples from each group. The arrows indicate the macrophages, the number of which was not significantly different among the groups. Original magnification $1000 \times$.

acute experiments, performed in healthy women, have shown an increased LF:HF ratio after red pepper ingestion ${ }^{(8)}$. Despite the beneficial effects of chronic treatment with capsaicin on cardiac autonomic control first shown by us, the sympathovagal balance did not change, as both groups that received capsaicin showed increased sympathetic modulation of the vascular tone, which was different only when capsaicin was administered in high doses.

Although paradoxical effects of improved autonomic modulation and increased sympathetic vascular modulation were observed, these did not influence arterial pressure and heart rate levels, results that are consistent with those reported earlier for humans who consumed red pepper for 4 weeks $^{(36)}$. However, after long periods of capsaicin treatment, decreased arterial pressure was observed in hypertensive patients ${ }^{(12)}$. These contradictory effects have been reported, and they are supported by the ability of capsaicin to activate an ion channel, a transient potential vanilloid type 1 channel, which controls the endothelial expression of neuropeptides involved in NO release ${ }^{(37)}$. These imbalances in the expression of neuropeptides possibly explain why capsaicin through its effects on blood pressure led to vasorelaxation in some studies $^{(38)}$, but to vasoconstriction in others ${ }^{(39)}$.

Rats with the MetS had higher aortic wall thickness when compared with non-obese and normotensive controls, but capsaicin did not induce these structural changes or cause a reduction in inflammation. This negative result is probably related to the short period of treatment, as other authors have reported benefits in mice fed a high-fat diet with capsaicin supplementation for 10 weeks ${ }^{(26)}$. Moreover, capsaicin could be less potent in reducing inflammatory processes, as atorvastatin has previously been reported to cause reduction in macrophage infiltration in diabetic rats ${ }^{(40)}$.

Capsaicinoids are powerful irritants, causing burning and pain at low concentrations on the skin and mucous membranes, and this could be a reason for the limitation of their use in daily practice. Given orally, they induce an increase in salivation and gastric secretion, a rapid change in sensation, and warm-to-intolerable burning, which is variably tolerated, depending on the individual ${ }^{(41)}$.

One important difference of the present study when compared with other studies was that capsaicin was orogastrically administered to the rats, adding strength to this part of the protocol. We were able to provide precise doses to the rats studied. We believe that in this way we excluded biases such as ad libitum intake and palatability and evaluated the effect of the 0.5 and $1.0 \mathrm{mg} / \mathrm{kg}$ BW doses for $14 \mathrm{~d}$. Clearly, longerduration studies could possibly contribute other information not provided here. Furthermore, to our knowledge, this is the first study in the literature to evaluate the effect of synthetic capsaicin in an animal model of the MetS.

We conclude that capsaicin cannot improve lipid and glucose abnormalities in an animal model of the MetS, but that this nutraceutical can exert beneficial cardiovascular effects. 
It is possible that the cardiovascular system is adapted to offset the acute increase in the sympathetic drive shown in other studies. This information opens an avenue to be explored, in future work, considering evaluation of the autonomic system in humans with the MetS given diets enriched with capsaicin

\section{Acknowledgements}

The authors thank Melissa Markoski (Laboratory of Molecular and Cellular Cardiology, Instituto de Cardiologia/ Fundação Universitária de Cardiologia, Brazil) for her technical assistance.

The present study was supported by Coordenação de Aperfeiçoamento de Pessoal de Nível Superior, Financiadora de Estudos e Projetos and Fundação de Amparo à Pesquisa do Estado do Rio Grande do Sul. They had no role in the design, analysis or writing of this article. B. D. S. was the recipient of a scholarship from $\mathrm{CNPq}$ (research productivity fellowship).

The authors' contributions are as follows: C. d. S. T., B. D. S., K. R. C. and L. M. participated in study concept and design; C. d. S. T. and B. D. S. participated in data search; C. d. S. T., K. R. C. and L. M. participated in data acquisition; C. d. S. T., B. D. S., K. R. C. and L. M. participated in data analysis and interpretation; B. D. S., K. R. C. and L. M. contributed to the statistical verification of the data; C. d. S. T., B. D. S., K. R. C. and L. M. contributed to the writing and revision of the report. All authors reviewed and approved the final version of the report.

The authors declare that they have no competing interests.

\section{References}

1. Mottillo S, Filion KB, Genest J, et al. (2010) The metabolic syndrome and cardiovascular risk: a systematic review and meta-analysis. J Am Coll Cardiol 56, 1113-1132.

2. Lehnen AM, Rodrigues B, Irigoyen MC, et al. (2013) Cardiovascular changes in animal models of metabolic syndrome. J Diabetes Res 2013, 1-11.

3. Leguisamo NM, Lehnen AM, Machado UF, et al. (2012) GLUT4 content decreases along with insulin resistance and high levels of inflammatory markers in rats with metabolic syndrome. Cardiovasc Diabetol 16, 1-10.

4. Richard D, Kefi K, Barbe U, et al. (2009) Weight and plasma lipid control by decaffeinated green tea. Pharmacol Res 59, 351-354.

5. Weisberg SP, Leibel R \& Tortoriello DV (2008) Dietary curcumin significantly improves obesity-associated inflammation and diabetes in mouse models of obesity. Endocrinology 149, 3549-3558.

6. Reyes-Escogido Mde L, Gonzalez-Mondragon EG \& Vazquez-Tzompantzi E (2011) Chemical and pharmacological aspects of capsaicin. Molecules 16, 1253-1270.

7. Kempaiah RK, Manjunatha H \& Srinivasan K (2005) Protective effect of dietary capsaicin on induced oxidation of low-density lipoprotein in rats. Mol Cell Biochem 275, 7-13.

8. Yoshioka M, St-Pierre S, Suzuki M, et al. (1998) Effects of red pepper added to high-fat and high-carbohydrate meals on energy metabolism and substrate utilization in Japanese women. Br J Nutr 80, 503-510.

9. Kang JH, Kim CS, Han IS, et al. (2007) Capsaicin, a spicy component of hot peppers, modulates adipokine gene expression and protein release from obese-mouse adipose tissues and isolated adipocytes, and suppresses the inflammatory responses of adipose tissue macrophages. FEBS Lett 581, 4389-4396.

10. Ma L, Zhong J, Zhao Z, et al. (2011) Activation of TRPV1 reduces vascular lipid accumulation and attenuates atherosclerosis. Cardiovasc Res 92, 504-513.

11. Scientific Committee on Food (2002) Opinion of the Scientific Committee on Food on Capsaicin. Brussels: European Commission, Scientific Committee on Food (SCF/CS/ FLAV/FLAVOUR/8 ADD1 Final).

12. Harada N \& Okajima K (2009) Effects of capsaicin and isoflavone on blood pressure and serum levels of insulin-like growth factor-I in normotensive and hypertensive volunteers with alopecia. Biosci Biotechnol Biochem 73, 1456-1459.

13. Li J \& Wang DH (2003) High-salt-induced increase in blood pressure: role of capsaicin-sensitive sensory nerves. J Hypertens 21, 577-582.

14. NIH (1996) Guide for the Care and Use of Laboratory Animals. Washington, DC: National Academy Press.

15. Kilkenny C, Browne WJ, Cuthill IC, et al. (2010) Improving bioscience research reporting: the ARRIVE guidelines for reporting animal research. J Pharmacol Pharmacother 1 , 94-99.

16. Bernardis LL (1970) Prediction of carcass fat, water and lean body mass from Lee's "nutritive ratio" in rats with hypothalamic obesity. Experientia 26, 789-790.

17. Geloneze B \& Tambascia M (2006) Laboratorial evaluation and diagnosis of insulin resistance. Arq Bras Endocrinol Metabol 50, 208-215.

18. Malliani A, Pagani M, Lombardi F, et al. (1991) Cardiovascular neural regulation explored in the frequency domain. Circulation 84, 482-492.

19. Montano N, Porta A, Cogliati C, et al. (2009) Heart rate variability explored in the frequency domain: a tool to investigate the link between heart and behavior. Neurosci Biobehav Rev 33, 71-80.

20. Stauss HM (2007) Identification of blood pressure control mechanisms by power spectral analysis. Clin Exp Pharmacol Physiol 34, 362-368.

21. Fazan R Jr, de Oliveira M, da Silva VJ, et al. (2005) Frequency-dependent baroreflex modulation of blood pressure and heart rate variability in conscious mice. Am J Physiol Heart Circ Physiol 289, 1968-1975.

22. Friedewald WT, Levy RI \& Fredrickson DS (1972) Estimation of the concentration of low-density lipoprotein cholesterol in plasma, without use of the preparative ultracentrifuge. Clin Chem 18, 499-502.

23. Lahiri MK, Kannankeril PJ \& Goldberger JJ (2008) Assessment of autonomic function in cardiovascular disease: physiological basis and prognostic implications. J Am Coll Cardiol 51, $1725-1733$.

24. Garruti G, Giampetruzzi F, Vita MG, et al. (2012) Links between metabolic syndrome and cardiovascular autonomic dysfunction. Exp Diabetes Res 2012, 1-9.

25. Manjunatha H \& Srinivasan K (2007) Hypolipidemic and antioxidant effects of dietary curcumin and capsaicin in induced hypercholesterolemic rats. Lipids 42, 1133-1142.

26. Kang JH, Tsuyoshi G, Han IS, et al. (2010) Dietary capsaicin reduces obesity-induced insulin resistance and hepatic steatosis in obese mice fed a high-fat diet. Obesity (Silver Spring) 18, 780-787.

27. Kawada T, Hagihara K \& Iwai K (1986) Effects of capsaicin on lipid metabolism in rats fed a high fat diet. J Nutr $\mathbf{1 1 6}$ $1272-1278$. 
28. Tsukahara F, Uchida Y, Ohba K, et al. (1998) The effect of acute cold exposure and norepinephrine on uncoupling protein gene expression in brown adipose tissue of monosodium glutamate-obese mice. Jpn J Pharmacol 77, 247-249.

29. Kirby RF, Sokoloff G, Perdomo E, et al. (1999) Thermoregulatory and cardiac responses of infant spontaneously hypertensive and Wistar-Kyoto rats to cold exposure. Hypertension 33, 1465-1469.

30. Cypess AM, Lehman S, Williams G, et al. (2009) Identification and importance of brown adipose tissue in adult humans. N Engl J Med 360, 1509-1517.

31. Cypess AM \& Kahn CR (2010) The role and importance of brown adipose tissue in energy homeostasis. Curr Opin Pediatr 22, 478-484

32. Dömötör A, Szolcsányi J \& Mózsik G (2006) Capsaicin and glucose absorption and utilization in healthy human subjects. Eur J Pharmacol 534, 280-283.

33. Ahuja KD, Robertson IK, Geraghty DP, et al. (2006) Effects of chili consumption on postprandial glucose, insulin, and energy metabolism. Am J Clin Nutr 84, 63-69.

34. Lehnen AM, Leguisamo NM, Casali KR, et al. (2013) Progressive cardiovascular autonomic dysfunction in rats with evolving metabolic syndrome. Auton Neurosci 176 $64-69$.
35. Ohnuki K, Moritani T, Ishihara K, et al. (2001) Capsaicin increases modulation of sympathetic nerve activity in rats: measurement using power spectral analysis of heart rate fluctuations. Biosci Biotechnol Biochem 65, 638-643.

36. Ahuja KD, Robertson IK, Geraghty DP, et al. (2007) The effect of 4-week chilli supplementation on metabolic and arterial function in humans. Eur J Clin Nutr 61, 326-333.

37. Szallasi A \& Blumberg PM (1999) Vanilloid (capsaicin) receptors and mechanisms. Pharmacol Rev 51, 159-212.

38. Yang D, Luo Z, Ma S, et al. (2010) Activation of TRPV1 by dietary capsaicin improves endothelium-dependent vasorelaxation and prevents hypertension. Cell Metab 12, 130-141.

39. Scotland RS, Chauhan S, Davis C, et al. (2004) Vanilloid receptor TRPV1, sensory C-fibers, and vascular autoregulation a novel mechanism involved in myogenic constriction. Circ Res 95, 1027-1034.

40. Linthout SV, Riad A, Dhayat N, et al. (2007) Anti-inflammatory effects of atorvastatin improve left ventricular function in experimental diabetic cardiomyopathy. Diabetologia 50, 1977-1986.

41. Govindarajan VS \& Sathyanarayana MN (1991) Capsicumproduction, technology, chemistry, and quality. Part V. Impact on physiology, pharmacology, nutrition, and metabolism; structure, pungency, pain, and desensitization sequences. Crit Rev Food Sci Nutr 29, 435-474. 